\title{
Periodic Solutions of Higher Order with Restoring Terms \\ Delay Functional Differential Equation with Neutral Type
}

\author{
Haiqing Wang \\ School of Science \\ Tianjin Polytechnic University \\ Tianjin 300160 China \\ E-mail: haiqingwang@tjpu.edu.cn
}

\begin{abstract}
Using the theory of coincidence degree, the authors have studied the existence of periodic solutions of a type of higher order with restoring terms delay functional differential equations with neutral type, and some new results for the existence of periodic solutions have been obtained.
\end{abstract}

Keywords: Delay functional differential equation, Coincidence degree, Periodic solutions

\section{Introduction and Lemma}

The functional differential equation with time delay, because of its wide application, has long been a main subject of common concern. But because the argument in complex time delay may depend on condition itself or variation rate, it is difficult to predict the property of the solution to the functional differential equation with complex deviating argument. It brings such big difficulty to our research work that in a long period of time the research of this equation progressed slowly. In recent years with further development of nonlinear functional analysis and algebra-ictopology, people have already had some achievement on the research of this equation. This paper mainly study periodic solutions of the following kind:

$x^{(m)}(t)+a(t) x^{(m)}(t-\tau)+f(x(t-\sigma)) \dot{x}(t-\sigma)+b(t) g(x(t-\tau))+c(t) x(t-\tau)=p(t)$

Lemma ${ }^{[7]}$ Let $X, Y$ be Banach space, $L: D_{L} \subset X \rightarrow Y$ is a Fredholm mapping of index $0 \quad P: X \rightarrow X, Q: Y \rightarrow Y$ are continuous mapping projector; $\Omega$ is an open bounded set in $X$,

and $N: \bar{\Omega} \times[0,1] \rightarrow Y$ is $L$-Compact on $\bar{\Omega}$, further more suppose:

(a) $L x \neq \lambda N(x, \lambda), \forall x \in D_{L} \cap \partial \Omega, \lambda \in(0,1)$;

(b) $Q N(x, 0) \neq 0, \forall x \in \operatorname{Ker}(L) \cap \partial \Omega$;

(c) $\operatorname{deg}(Q N(x, 0), \operatorname{Ker}(L) \cap \Omega, 0) \neq 0$.

then the equation $L x=N(x, 1)$ has at least one solution in $\bar{\Omega}$. Where deg is Brouwer degree.

\section{Main Results and Proof of Theorems}

Theorem: Suppose $f, a, b, c, g, p$ are continuous for their variables respectively; There exits $T$, such that $p(t+T)=p(t), a(t+T)=a(t), b(t+T)=b(t)>0,0<|a(t)|<a_{1}<1$,

$c(t+T)=c(t)<0, \quad a_{m}=\max _{t \in R}\left\{\left|a^{m}(t)\right|\right\}<c_{0}=\min _{t \in R}\{|c(t)|\} \leq c_{1}=\max _{t \in R}\{|c(t)|\}<\frac{1-a_{1}}{T^{m}}$,

$a(t) \in C^{m}(R, R)$ and further more suppose are as follows,

a. $\exists 0<M<\frac{1-a_{1}-c_{1} T^{m}}{T^{m-1}}, \forall x \in R, \quad|f(x)| \leq M$,

b. $\exists A>0, \forall x \in \boldsymbol{R}, \quad|g(x)| \leq A$;

c. $\forall 0 \neq x \in \boldsymbol{R}, \quad x g(x)<0$

then Eq.(1)has at least one T-periodic solution. 
Proof of Theorem : In order to use continuation theorem to obtain T-periodic solution of Eq.(1), we firstly make some required preparations. Let $X=\left\{x \in C^{m-1}(\boldsymbol{R}, \boldsymbol{R}) \mid x(t+T)=x(t)\right\}$

$Y=\{y \in C(\boldsymbol{R}, \boldsymbol{R}) \mid y(t+T)=y(t)\}$ and the norm of $X$ is $\|x\|=\max _{0 \leq i \leq m-1}\left\{\left|x^{(i)}\right|_{\infty}\right\}$, there $\left|x^{(0)}\right|_{\infty}$

$=|x|_{\infty}=\max _{t \in R}\{|x(t)|\},\left|x^{(i)}\right|_{\infty}=\max _{t \in R}\left\{\left|x^{(i)}(t)\right|\right\}, \quad i=1,2, \cdots, m-1,\|y\|=\max _{t \in R}\{|y(t)|\}$, then the $X$ and $Y$ with this norm are Banach space.

Firstly, we study the priori bound of T-periodic solution of the following equation

$$
x^{(m)}(t)+\lambda a(t) x^{(m)}(t-\tau)+\lambda f(x(t-\sigma)) \dot{x}(t-\sigma)+\lambda b(t) g(x(t-\tau))+\lambda c(t) x(t-\tau)=\lambda^{2} p(t)
$$

Suppose that $x=x(t) \in X$ is an arbitrary T-periodic solution of Eq.(1.1), put $x(t)$ into (1.1) and then integrate both sides of $(1.1)$ on $[0, T]$, so yield

$$
\int_{0}^{T}\left[a(t) x^{(m)}(t-\tau)+b(t) g(x(t-\tau))+c(t) x(t-\tau)\right] d t=\lambda \int_{0}^{T} p(t) d t
$$

we easily get :

$(-1)^{m} \int_{0}^{T} a^{(m)}(t) x(t-\tau) d t+\int_{0}^{T} b(t) g(x(t-\tau)) d t+\int_{0}^{T} c(t) x(t-\tau) d t=\lambda \int_{0}^{T} p(t) d t$

so there exits a $t_{0} \in[0, T]$, such that:

$(-1)^{m} a^{(m)}\left(t_{0}\right) x\left(t_{0}-\tau\right)+b\left(t_{0}\right) g\left(x\left(t_{0}-\tau\right)\right)+c\left(t_{0}\right) x\left(t_{0}-\tau\right)=\lambda p\left(t_{0}\right)$

i.e. $\left|c\left(t_{0}\right) x\left(t_{0}-\tau\right)\right|=\left|\lambda p\left(t_{0}\right)-(-1)^{m} a^{(m)}\left(t_{0}\right) x\left(t_{0}-\tau\right)-b\left(t_{0}\right) g\left(x\left(t_{0}-\tau\right)\right)\right|$

so $\left|c_{0} x\left(t_{0}-\tau\right)\right| \leq p_{1}+a_{m}\left|x\left(t_{0}-\tau\right)\right|+b_{1} A$

i.e. $\left|x\left(t_{0}-\tau\right)\right| \leq \frac{b_{1} A+p_{1}}{c_{0}-a_{m}} \underline{\Delta} A_{1}$ where $b_{1}=\max _{t \in R}\{|b(t)|\}, \quad p_{1}=\max _{t \in R}\{|p(t)|\}$

let $t_{0}-\tau=n T+t_{1}, \quad n \in N, t_{1} \in[0, T]$, so $\left|x\left(t_{1}\right)\right|=\left|x\left(t_{0}-\tau\right)\right| \leq A_{1}$

In view of $\forall t \in[0, T], x(t)=x\left(t_{1}\right)+\int_{t_{1}}^{t} \dot{x}(s) d s$, we have

$$
\begin{gathered}
|x(t)|=\left|x\left(t_{1}\right)+\int_{t_{1}}^{t} \dot{x}(s) d s\right| \leq A_{1}+\int_{t_{1}}^{t}|\dot{x}(s)| d s \leq A_{1}+\int_{0}^{T}|\dot{x}(t)| d t \\
\text { i.e. }\left|x^{(0)}\right|_{\infty}=|x|_{\infty} \leq A_{1}+\int_{0}^{T}|\dot{x}(t)| d t
\end{gathered}
$$

Noting $x(t)=x(t+T)$, so there must exist numbers $\xi_{i} \in(0, T)$, such that $x^{(i)}\left(\xi_{i}\right)=0$, there $i=1,2,3, \cdots, m-1$.

For $\forall t \in[0, T], x^{(i)}(t)=x^{(i)}\left(\xi_{i}\right)+\int_{\xi_{i}}^{t} x^{(i+1)}(s) d s=\int_{\xi_{i}}^{t} x^{(i+1)}(s) d s$, we have

$$
\begin{aligned}
& \left|x^{(i)}(t)\right|=\left|\int_{\xi_{i}}^{t} x^{(i+1)}(s) d s\right| \leq \int_{0}^{T}\left|x^{(i+1)}(t)\right| d t \leq T \int_{0}^{T}\left|x^{(i+2)}(t)\right| d t \\
& \leq T^{2} \cdot \int_{0}^{T}\left|x^{(i+3)}(t)\right| d t \leq \cdots \leq T^{m-(i+1)} \int_{0}^{T}\left|x^{(i+m-i)}(t)\right| d t=T^{m-(i+1)} \int_{0}^{T}\left|x^{(m)}(t)\right| d t,
\end{aligned}
$$

$$
\text { i.e. } \quad\left|x^{(i)}\right|_{\infty} \leq T^{m-(i+1)} \int_{0}^{T}\left|x^{(m)}(t)\right| d t, \quad i=1,2, \cdots, m-1
$$

Combining (1.2),(1.3), we get

$$
\left|x^{(0)}\right|_{\infty}=|x|_{\infty} \leq T^{m-1} \int_{0}^{T}\left|x^{(m)}(t)\right| d t
$$

By (1.1), we get

$\int_{0}^{T}\left|x^{(m)}(t)\right| d t \leq \int_{0}^{T}\left|-\lambda a(t) x^{(m)}(t-\tau)-\lambda f(x(t-\sigma)) \dot{x}(t-\sigma)\right| d t+\int_{0}^{T}\left|-\lambda b(t) g(x(t-\tau))-\lambda c(t) x(t-\tau)+\lambda^{2} p(t)\right| d t$

Combining the condition a and $\mathrm{b}$, we easily obtain

$\int_{0}^{T}\left|x^{(m)}(t)\right| d t \leq a_{1} \int_{0}^{T}\left|x^{(m)}(t-\tau)\right| d t+M \int_{0}^{T}|\dot{x}(t-\sigma)| d t+c_{1} \int_{0}^{T}|x(t-\tau)| d t+T A b_{1}+T p_{1}$ 


$$
\text { i.e. } \int_{0}^{T}\left|x^{(m)}(t)\right| d t \leq a_{1} \int_{0}^{T}\left|x^{(m)}(t)\right| d t+M T|\dot{x}|_{\infty}+c_{1} T|x|_{\infty}+T A b_{1}+T p_{1}
$$

Noting (1.3), (1.4) and (1.5), we observe

$$
\begin{gathered}
\int_{0}^{T}\left|x^{(m)}(t)\right| d t \leq a_{1} \int_{0}^{T}\left|x^{(m)}(t)\right| d t+M T \cdot T^{m-2} \int_{0}^{T}\left|x^{(m)}(t)\right| d t+c_{1} T\left(A_{1}+T^{m-1} \int_{0}^{T}\left|x^{(m)}(t)\right| d t\right)+T A b_{1}+T p_{1} \\
\left(1-a_{1}-M T^{m-1}-c_{1} T^{m}\right) \int_{0}^{T}\left|x^{(m)}(t)\right| d t \leq T A b_{1}+T p_{1}+c_{1} T A_{1} \\
\text { so } \int_{0}^{T}\left|x^{(m)}(t)\right| d t \leq \frac{T A b_{1}+T p_{1}+c_{1} T A_{1}}{1-a_{1}-M T^{m-1}-c_{1} T^{m}}
\end{gathered}
$$

Noting(1.3),(1.5)and(1.6), we have

$$
\begin{aligned}
& \left|x^{(0)}\right|_{\infty}=|x|_{\infty} \leq A_{1}+T^{m-1} \cdot \frac{T A b_{1}+T p_{1}+c_{1} T A_{1}}{1-a_{1}-M T^{m-1}-c_{1} T^{m}}=A_{1}+\frac{T^{m}\left(A b_{1}+p_{1}+c_{1} A_{1}\right)}{1-a_{1}-M T^{m-1}-c_{1} T^{m}}=\Delta \omega_{0} \\
& \left|x^{(i)}\right|_{\infty} \leq T^{m-(i+1)} \cdot \frac{T A b_{1}+T p_{1}+c_{1} T A_{1}}{1-a_{1}-M T^{m-1}-c_{1} T^{m}}=\frac{T^{m-i}\left(A b_{1}+p_{1}+c_{1} A_{1}\right)}{1-a_{1}-M T^{m-1}-c_{1} T^{m}}=\Delta \omega_{i} i=1,2, \cdots, m-1
\end{aligned}
$$

Let $\omega=\max _{0 \leq i \leq m}\left\{\omega_{i}+1\right\}$, and we take $\Omega=\{x \mid x \in X:\|x\|<\omega\}$, then $\Omega$ is an open and bounded set in $X$.

Let $L: D_{L} \subset X \rightarrow Y: x \rightarrow L x=x^{(m)}(t)$;

$N: X \times I \rightarrow Y: x \rightarrow N(x, \lambda)=-a(t) x^{(m)}(t-\tau)-f(x(t-\sigma)) \dot{x}(t-\sigma)-b(t) g(x(t-\tau))-c(t) x(t-\tau)+\lambda p(t)$, then the corresponding equation of $L x=\lambda N(x, \lambda)$ is Eq.(1.1).

Now, we define projection operators as follows,

$$
P: X \rightarrow \operatorname{Ker}(L): x \rightarrow P x=\frac{1}{T} \int_{0}^{T} x(t) d t ; Q: Y \rightarrow Y / \operatorname{Im}(L): y \rightarrow Q y=\frac{1}{T} \int_{0}^{T} y(t) d t
$$

obviously, $P, Q$ are continuous operator, $\operatorname{Im}(P)=R=\operatorname{Ker}(L), \operatorname{Ker}(Q)=\operatorname{Im}(L)$, and it is easy to prove that $L$ is a Fredholm mapping of index 0 and $N$ is $L$-Compact on $\bar{\Omega}$.

From the above discussion and the construction of $\Omega$, we have known that $\forall x \in D_{L} \cap \partial \Omega$ $\lambda \in(0,1)$, therefore the condition (a) of lemma holds.

For arbitrary $x \in \operatorname{Ker}(L) \cap \partial \Omega,\|x\|=\omega$, by the definition of $Q, N$, we have

$$
\begin{aligned}
& Q N(x, 0)=\frac{1}{T} \int_{0}^{T}\left[-a(t) x^{(m)}(t-\tau)-f(x(t-\sigma)) \dot{x}(t-\sigma)-b(t) g(x(t-\tau))-c(t) x(t-\tau)\right] d t \\
& =-\frac{1}{T} \int_{0}^{T} b(t) g(x(t-\tau)) d t-\frac{1}{T} \int_{0}^{T} c(t) x(t-\tau) d t \\
& \text { so } x Q N(x, 0)=-\frac{1}{T} x \int_{0}^{T} b(t) g(x(t-\tau)) d t-\frac{1}{T} x \int_{0}^{T} c(t) x(t-\tau) d t \\
& =-\frac{1}{T} x g(x) \int_{0}^{T} b(t) d t-\frac{1}{T} x^{2} \int_{0}^{T} c(t) d t>0
\end{aligned}
$$

therefore the condition (b) of lemma holds.

Make a transformation $H(x, \mu)=\mu x+(1-\mu) Q N(x, 0), \forall x \in \partial \Omega \cap \operatorname{Ker}(L), \mu \in[0,1]$

We have $x H(x, \mu)=\mu x^{2}+x(1-\mu) Q N(x, 0)$

$=\mu x^{2}-(1-\mu) \frac{1}{T} g(x) x \int_{0}^{T} b(t) d t-(1-\mu) \frac{1}{T} x^{2} \int_{0}^{T} c(t) d t>0$

so $H(x, \mu) \neq 0$, i.e. $H(x, \mu) \neq 0$ is a homotopy, $\operatorname{deg}(Q N(x, 0), \operatorname{Ker}(L) \cap \Omega, 0)=\operatorname{deg}(I$,

$\operatorname{Ker}(L) \cap \Omega, 0)=\operatorname{deg}(I, R \cap \Omega, 0) \neq 0$, where $I$ is identity mapping and the condition (c) of lemma holds.

From above all, the requirements of lemma are all met, so Eq. (1)has at least one T-periodic solution under the condition of theorem 1, so far the proof of theorem is completed.

\section{References}

Gaines R E and Mawhin J L. Coincidence degree and nonlinear differential equations [c]. Lecture 
Notes Math, springer-verlag, 1977, 568

Liu Xiping, Jia Mei and Ge Weigao. Periodic solutions to a type of Duffing equation with complex deviating argument[J],Appl. Math. J. Chinese Univ. Ser. A 2003,18(1):51-56

Mari P O and Zanolin F. Boundary value problems for forced nonlinear equations at resonance.

Lecture Notes in Math,1151. Ordinary and Partial Differential Equation. Berlin: springer-verlag, 1984, 285 294

Mawhin J and Ward J J R. Nonuniform nonresonance conditions at the two first eigenvalues for forced periodic solutions of forced Linéard and Duffing equations. Rocky Mountain J Math, 1982,12(4):643 654

Pascale E, and Iannacci R. Periodic solution of a generalized Liénard equation with delay,. Lecture Notes Math, 1017. Berlin: Springer-Verlag, 1983, 148 156

Xiang Zigui, Liu Changmao, and Huang Xiankai. On Periodic Solutions of Delay Li é nard Equations[J], Journal of Jishou University (National Science Edition), 1998,19(4):35-40

Zheng Zuxiu, Theory of Functional Differential Equation [M].Hefei: Anhui education press, 1994 